\title{
Functionally substituted arylhydrazones as building blocks in heterocyclic synthesis: routes to pyridazines and pyridazinoquinazolines
}

\author{
Said Ahmed Soliman Ghozlan, Ismail Abdelshafy Abdelhamid,* \\ and Mohamed Hilmy Elnagdi* \\ Department of Chemistry, Faculty of Science, Cairo University, Giza, A. R. Egypt \\ E-mail:shelmy@access.com.eg, ismail_shafy@yahoo.com
}

\begin{abstract}
The arylhydrazones 2a-c were prepared via coupling acetoacetic acid with aromatic diazonium salts. These arylhydrazones condensed with ethyl cyanoacetate and malononitrile to yield the acyclic product 4 which cyclised only after long reflux into the pyridazines 5 or 6,11dihydropyridazino[1,6- $a$ ] quinazoline-4-carbonitrile $\mathbf{6}$ depending on the nature of substituent on the aryl moiety. Compound $\mathbf{2 b}$ and $\mathbf{2 c}$ reacted with $\alpha, \beta$-unsaturatednitriles $\mathbf{7}$ to yield the pyridazinoquinazoline $\mathbf{1 3}$ and $\mathbf{1 6}$ respectively.
\end{abstract}

Keywords: Azaenamines, pyridazines, pyridazinoquinazolines

\section{Introduction}

The chemistry and pharmacology of pyridazines has recently received considerable interest. This can be readily realized from vast number of papers and patents dealing with synthesis ${ }^{1-5}$, chemistry ${ }^{6-8}$, and biological activities of pyridazine derivatives ${ }^{9,10}$. In the last few years we have been involved in a programme aimed at developing efficient syntheses of pyridazines and fused pyridazines ${ }^{11-14}$ utilizing functionally substituted arylhydrazones precursors. In conjunction of this work we report here routes to pyridazinones, and pyridazinoquinazolines utilizing the readily obtainable 2a-c as starting materials.

\section{Results and Discussion}

Coupling acetoacetic acid with the diazonium salts 1a-c has afforded the corresponding pyruvaldehyde arylhydrazones $\mathbf{2 a - c}$ in $74-90.8 \%$ yields. Although it seemed logical to assign to syn structure 3 for these compounds based on observed low field NH signal that is attributable to 
deshielding by hydrogen bonding, X-ray crystal structure of 2a (figure 1) indicated that it exists in the antiform 2a. The low field hydrazone $\mathrm{NH}$ at $\delta 12 \mathrm{ppm}$ is not thus due to deshielding by hydrogene bonding as has been claimed earlier but most likely resulted from delocalization of nitrogen lone pair (C.f. Scheme 1 structure 2a(II)). Although, possible hydrogen bonding of hydrazone NH with DMSO cannot be overlooked.

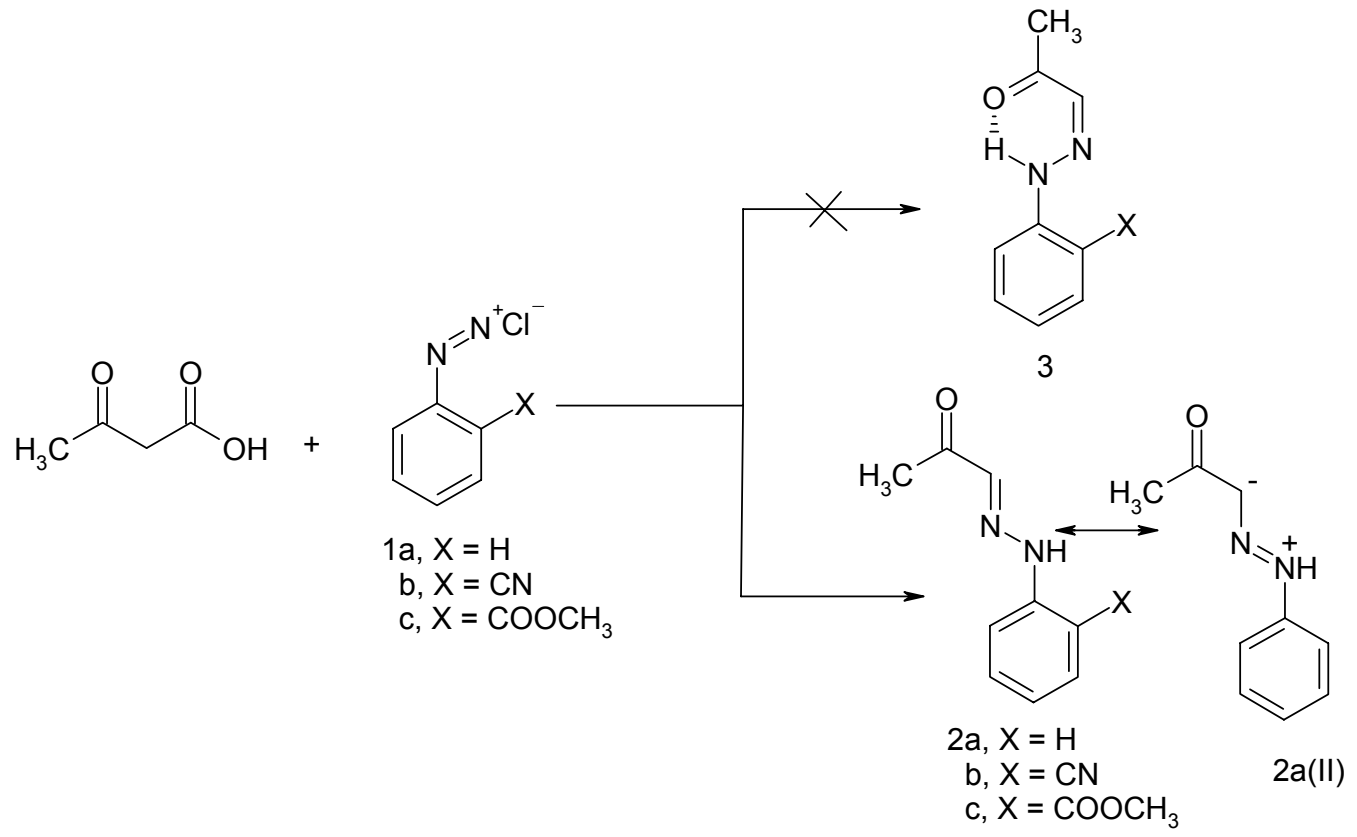

Scheme 1

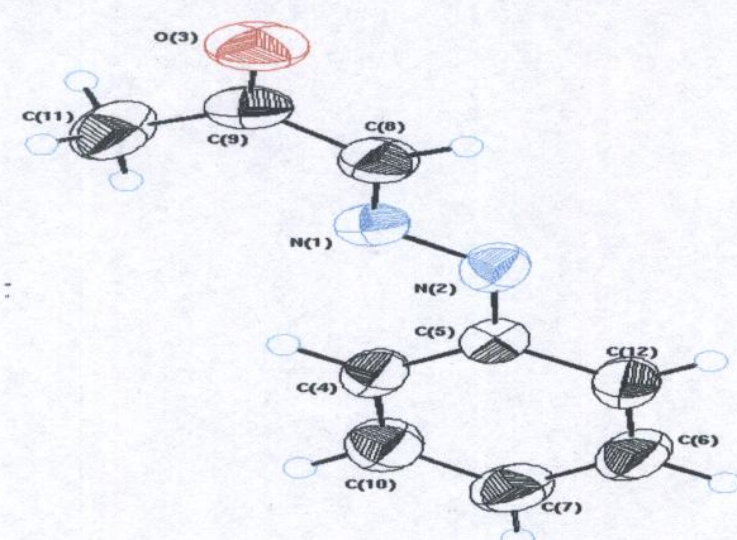

Figure 1. X-ray structure of compound 2a 
Table 1. Selected bond lengths and bond angles for compound 2a

\begin{tabular}{llll}
\hline Bond & Bond length & Bond & Bond angle \\
\hline N1-N2 & 1.335 & N2-N1-C8 & 117.8 \\
N1C8 & 1.285 & N1-N2-C5 & 121.6 \\
N2-C5 & 1.395 & N2-C5-C4 & 122.4 \\
C8-C9 & 1.450 & O3-C9-C8 & 118.9
\end{tabular}

Compound $\mathbf{2 b}$ condensed with ethyl cyanoacetate 3a to yield the acyclic condensation product $\mathbf{4 b}$ which cyclised into pyridazine $\mathbf{5 b}$ only on long reflux in acetic acid. This is in contrast to the behavior of $\mathbf{2} \mathbf{a}$, where the acyclic intermediate $\mathbf{4 a}$ could never be isolated, on reacting it with $\mathbf{3 a}{ }^{[15]}$. In contrast to the behavior of $\mathbf{2 a}, \mathbf{b}$, compound $\mathbf{2} \mathbf{c}$ condenses with ethyl cyanoacetate to yield acyclic product $\mathbf{4 c}$, which failed to cyclise into pyridazine $\mathbf{5 c}$ under a variety of conditions. The difference in behavior between $\mathbf{2 a , b}$ and $\mathbf{2 c}$ toward ethyl cyanacetate is believed to result from interaction with the ester function and hydrazone nitrogen which is involved in hydrogen bonding with ester function. ${ }^{1} \mathrm{H}$ NMR indicates that $\mathbf{4 b}, \mathbf{c}$ exist as a mixture of cis and trans forms I and II. Thus two overlapping triplets for $\mathrm{CH}_{3}$ ester and two overlapping quartets for $\mathrm{CH}_{2}$ were observed. Aromatic protons revealed expected pattern. Azomethine $\mathrm{CH}$ appeared as two signals and it is difficult to assign which $\mathrm{CH}$ for which isomer as complex factors are involved in this situation. Compound $\mathbf{2 c}$ also condensed with malononitrile $\mathbf{3 b}$ to yield the acyclic product $\mathbf{4 d}$ which then cyclises into pyridazinooquinazoline derivative $\mathbf{6}$ via intermediacy of $\mathbf{5} \mathbf{d}$.

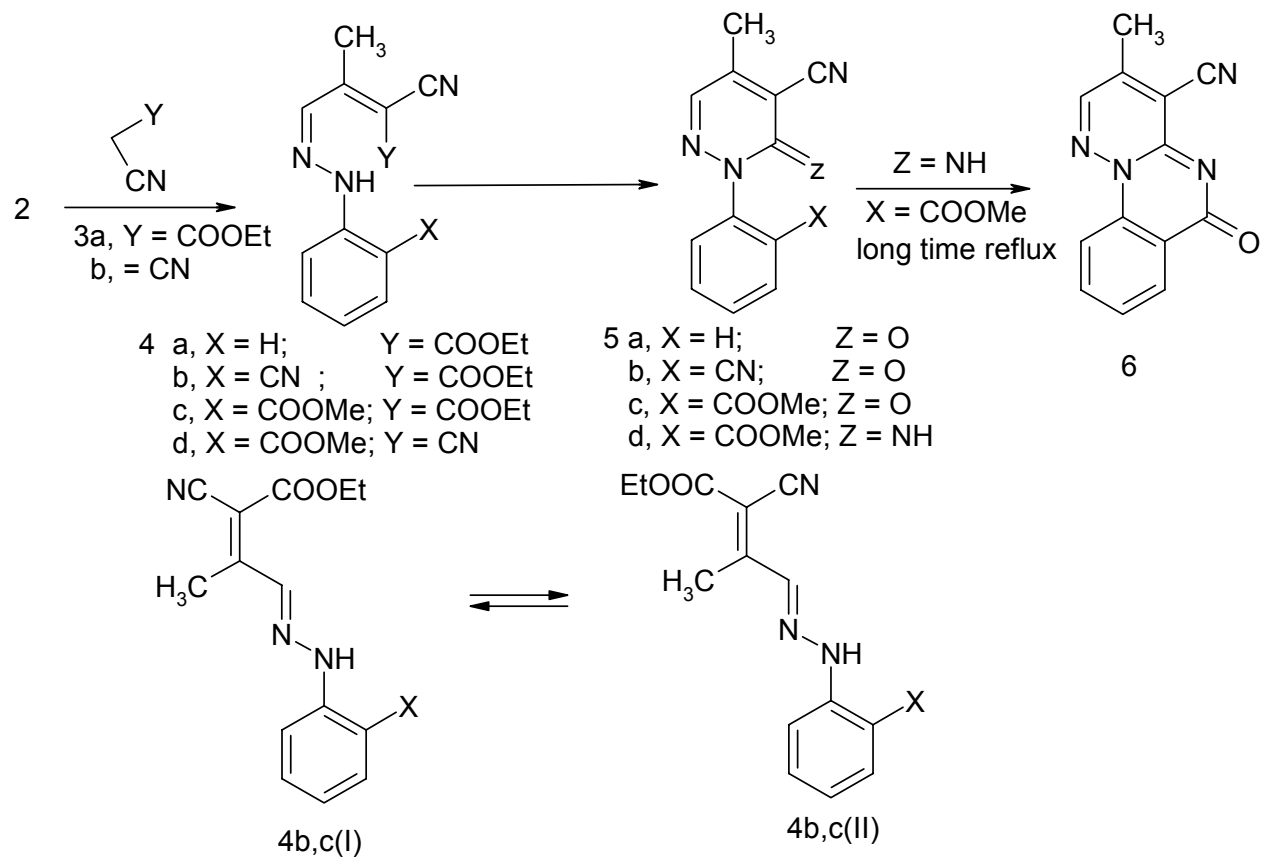

\section{Scheme 2}


Compounds 5a,b react readily with $\alpha$-substituted cinnamonitriles $7 \mathbf{a}-\mathbf{c}$ to yield acyclic intermediate $\mathbf{8}$ that readily cyclized into phthalazine derivatives 9a-c via hydrogen cyanide elimination. These same products were obtained from reaction of compounds $\mathbf{4 b} \mathbf{b}-\mathbf{d}$ with $\alpha$ substituted cinnamonitriles. It is believed that compounds $\mathbf{4}$ are initially cyclised into pyridazines 5 under reaction conditions which then reacts with $\alpha$-cinnamonitriles to give the targeted molecule. (C.f. scheme 3)

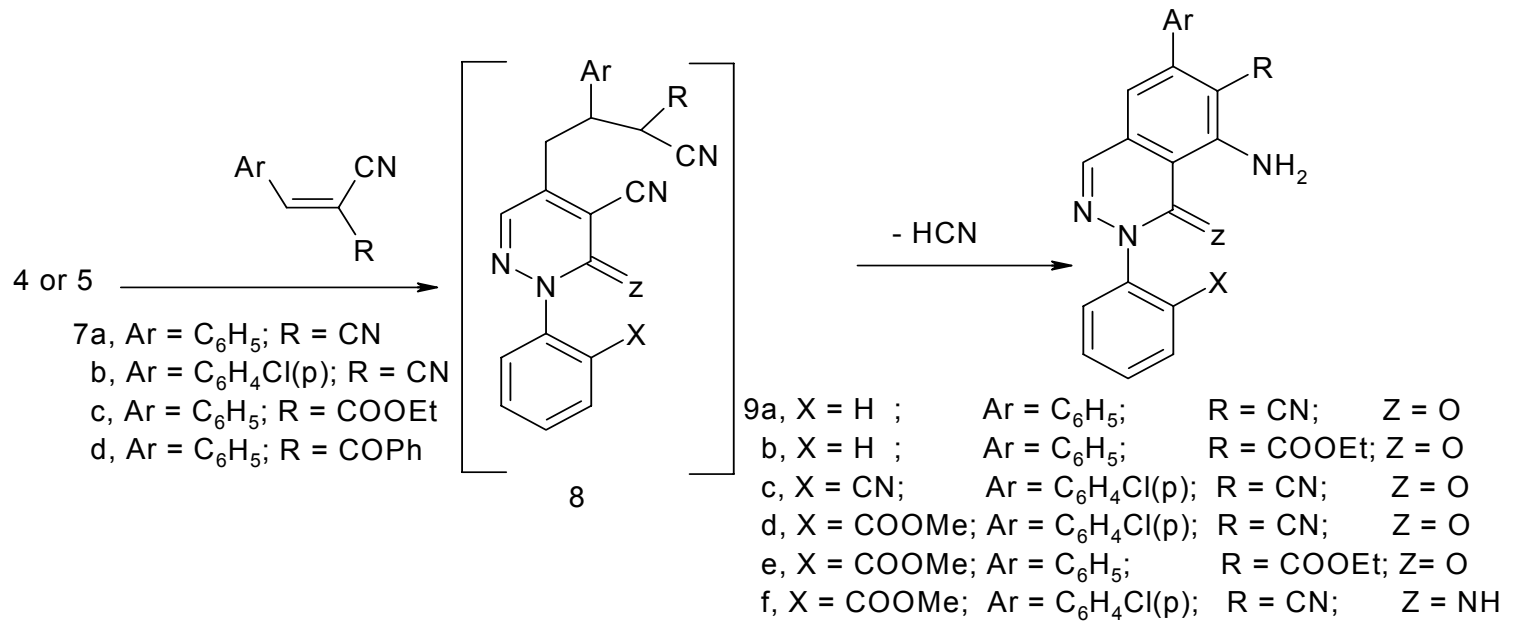

\section{Scheme 3}

It has recently been shown that aldehydehydrazone $\mathrm{CH}$ is electron rich and reacts with electrophiles ${ }^{16-18}$. Although it was proposed that presence of electron donating substituents at hydrazone nitrogen is necessary to maintain reactivity, we could show very recently that aryl hydrazones are also sufficiently reactive toward electrophiles under relatively mild conditions ${ }^{18}$. The reactivity of $\mathbf{2 a}$ toward $\alpha, \beta$-unsaturated nitriles has recently been utilized by us to construct a new synthetic approach for 1,4-dihydro-6-aminopyridazines ${ }^{13}$. In the present work we would like to show that even $\mathbf{2 b , c}$ with electron attracting substituents on aryl moiety are also reactive toward electrophiles. Thus reacting $\mathbf{2 b}$ with p-chlorobezylidnemalononitrile $\mathbf{7 b}$, ethyl benzylidenecyanoacetat 7c, and 2-benzoyl-3-phenylacrylonitrile 7d has resulted in formation of 1:1 adduct that can in theory be assigned structures 10-13. Structure 13a was readily established based on ${ }^{13} \mathrm{C}$ NMR that revealed presence of methyl signal at $\delta 25.21 \mathrm{ppm}$ and only one cyano carbon at $\delta 112.08 \mathrm{ppm}$. The structures of $\mathbf{1 3 b}, \mathbf{c}$ were elucidated based on IR and NMR where IR spectra revealed the absence of $\mathrm{CN}$ signals We believe that initially $\mathbf{1 1}$ is formed and cyclized into 12 which then cyclized into 13. (C.f. Scheme 4). 


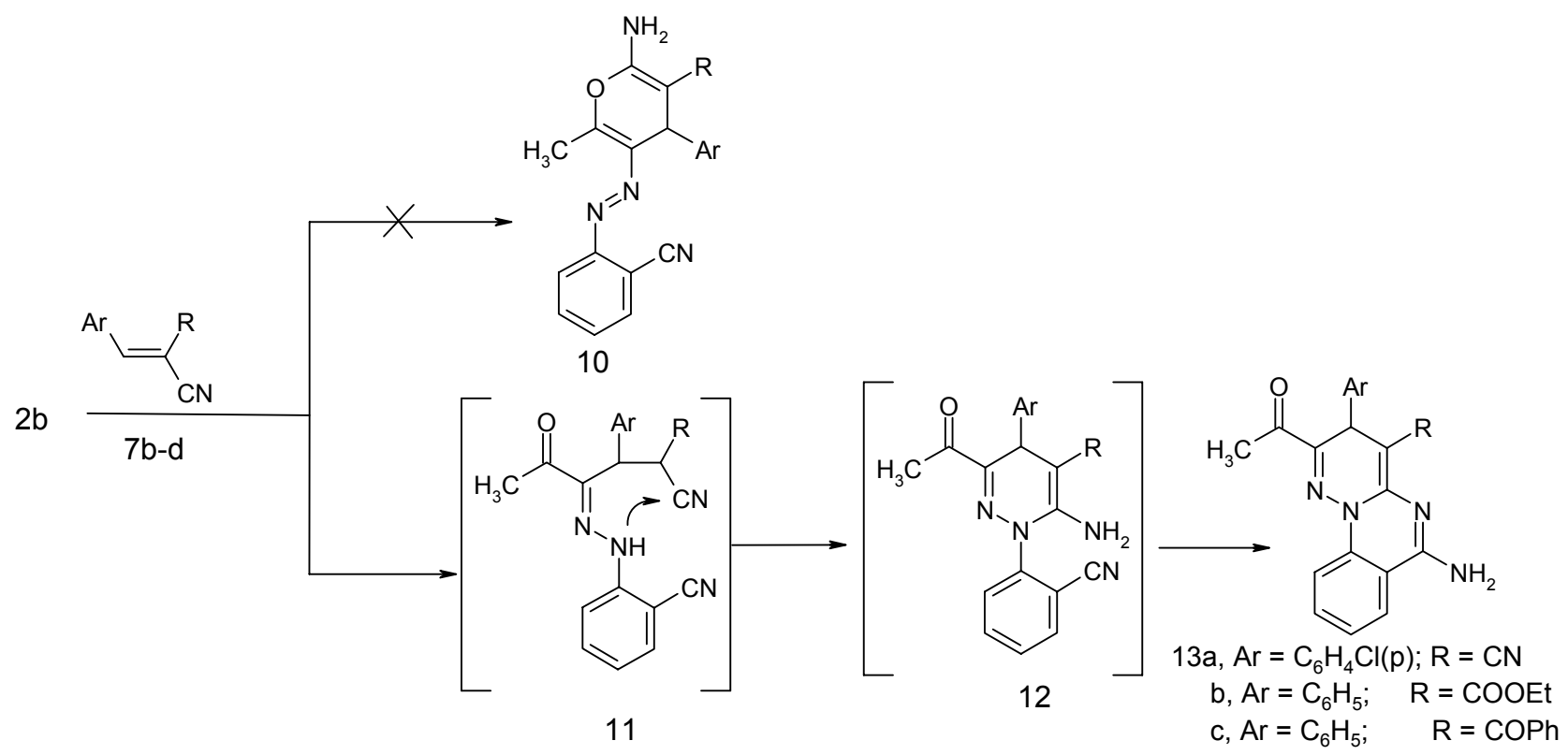

\section{Scheme 4}

In contrast to this $\mathbf{2 c}$ reacted with $\mathbf{7 b}$ to yield a product $\mathbf{1 4}$ that could not be identified. On the other hand, the reaction of $\mathbf{2 c}$ with $\mathbf{7 c}$ results in the formation of product of cyclization with methanol elimination to give compound 16. The structure of 16 was elucidated based on IR which indicated the absence of $\mathrm{CN}$ band, ${ }^{1} \mathrm{H}$ NMR which indicated the absence of $\mathrm{OCH}_{3}$ signal, and ${ }^{13} \mathrm{C}$ NMR spectra which indicated the absence of any $\mathrm{CN}$ and $\mathrm{OCH}_{3}$ bands and signals. (C.f. scheme 5).

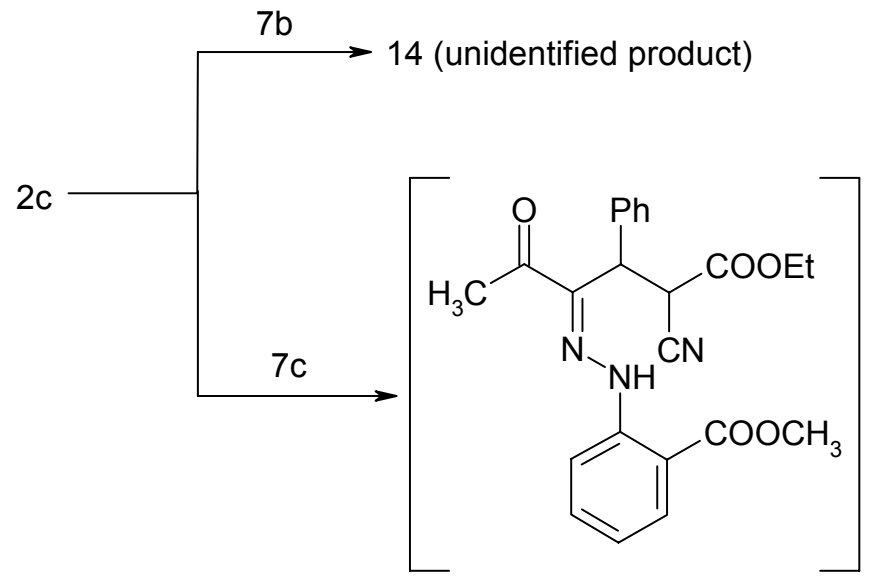

15<smiles>CCOC(=O)C1=C2NC(=O)c3ccccc3N2N=C(C(C)=O)C1c1ccccc1</smiles>

16

\section{Scheme 5}




\section{Conclusions}

We could show that Pyruvaldehyde-1-arylhydrazones 2a-c are valuable precursors to polyfunctionally substituted pyridazines, and pyridazinoquinazoline, moreover we could clearly show that hydrazone $\mathrm{CH}$ in $\mathbf{2}$ is activated toward electrophiles and thus can undergo Michael addition to $\alpha$-substituted cinnamonitriles under mild conditions.

\section{Experimental Section}

General Procedures. All melting points were determined on a Stuart melting point apparatus and are uncorrected. The ${ }^{1} \mathrm{H}$ and ${ }^{13} \mathrm{CNMR}$ spectra $(300 \mathrm{MHz})$ were recorded on Varian Gemini NMR spectrometer. Chemical shifts $(\delta)$ are given from TMS (0 ppm) as internal standard for ${ }^{1} \mathrm{H}-$ NMR and ${ }^{13} \mathrm{C}-\mathrm{NMR}$. Mass spectra were measured on a Shimadzu GMMS -QP-1000 EX mass spectrometer at $70 \mathrm{eV}$. The elemental analyses were performed at the micro analytical center, Cairo University. The IR spectra were recorded in KBr using a FTIR unit Bruker-vector 22 spectrophotometer. The crystal structure was determined by the X-ray unit at the National Research Center, Dokki, Cairo.

Crystallographic analysis for compound 2a. The crystals were mounted on a glass fiber. All measurements were performed on an ENRAF NONIUNS FR 590. The data were collected at temperature $20 \pm 1{ }^{\circ} \mathrm{C}$ using the $\omega$ scanning technique to a maximum of 20 of $27.12^{\circ}$. The temperature was solved by direct method using SIR 92 and full-matrix least squares. Nonhydrogen atoms were refined anisotropically. Hydrogen atoms were located geometrically and were refined isotropically.

Crystal data. $\mathrm{C}_{9} \mathrm{H}_{10} \mathrm{~N}_{2} \mathrm{O}, \mathrm{M}=162.19$, monoclinic, $a=5.4337$ (2), $b=10.1221$ (5), $\mathrm{c}=16.1178$ (10) $\AA, \alpha=\gamma=90.00^{\circ}, \beta=10 .(18) * 10^{10}$ space group: $\mathrm{P} 2_{1} / \mathrm{c} . \mathrm{Z}=4, \mathrm{D}_{\mathrm{x}}=1.236 \mathrm{Mg} \mathrm{m}^{-3}$, reflection 871 measured, $\theta_{\max }=25.03^{\circ}, \omega \mathrm{R}$ factor $=0.108$

General procedures for preparation of arylhydrazonopropan-2-one (2) ${ }^{\text {ref. }}{ }^{19} \mathrm{~A}$ mixture of $\mathrm{KOH}(3.5 \mathrm{~g})$ in $100 \mathrm{ml}$ of water, and $6.5 \mathrm{ml}$ of ethyl acetoacetate was allowed to stir at room temperature for 24 hours. This solution is then cooled at $0{ }^{\circ} \mathrm{C}$ temperature and then acidified with $4.5 \mathrm{ml}$ of Conc..HCl in $15 \mathrm{ml}$ of ice water. The resulting solution is the treated with aryldiazonium chloride (prepared from the corresponding aromatic amine $(0.05 \mathrm{~mol})$ and the appropriate quantities of both $\mathrm{HCl}$ and sodium nitrite). The mixture is made basic by addition of $8.0 \mathrm{~g}$ of sodium acetate. The solid product, so formed was collected by filtration.

(Phenyl-hydrazono)-propan-2-one (2a). ${ }^{\text {ref. }}{ }^{19}$ Yield $1.2 \mathrm{~g}(74 \%)$ as a solid, which was recrystallized from toluene to give yellow crystals (m.p 148-150) ${ }^{\circ} \mathrm{C}$ [lit. m.p. $=148{ }^{\circ} \mathrm{C}$; ; Anal. Calcd. for $\mathrm{C}_{9} \mathrm{H}_{10} \mathrm{~N}_{2} \mathrm{O}$ (162.19): C, 66.65; H, 6.21; N, 17.27. Found: C, 66.43; H, 6.05; N, 17.15. IR (KBr, cm ${ }^{-1}$ ): $3249.6(\mathrm{NH}), 1649.58(\mathrm{CO}) ;{ }^{1} \mathrm{H}$ MNR (300 MHz, DMSO-d6): $\delta$, ppm: 2.32 (s, $\left.3 \mathrm{H}, \mathrm{CH}_{3}\right), 6.93$ (s, 1H, vinyl-H), 6.95-7.339 (m, 5H, Ph-H), 11.33 (s, 1H, NH).;MS (EI): m/z (\%) $=162\left(\mathrm{M}^{+}\right)$. 
2-[N"-(2-Oxo-propylidene)-hydrazino]-benzonitrile (2b). Yield $1.4 \mathrm{~g}(74.8 \%)$ as a solid, which was recrystallized from from ethanol as white solid (m.p: 122-124 $\left.{ }^{\circ} \mathrm{C}\right)$. Anal. Calcd. for $\mathrm{C}_{10} \mathrm{H}_{9} \mathrm{~N}_{3} \mathrm{O}$ (187.20): C, 64.16; H, 4.97; N, 22.45. Found: C, 64.45; H, 4.92; N, 22.67. IR (KBr, $\mathrm{cm}^{-1}$ ): $3256.8(\mathrm{NH}), 2222(\mathrm{CN}), 1675(\mathrm{CO}) .{ }^{1} \mathrm{H}$ MNR (300 MHz, DMSO-d6): $\delta$, ppm 2.36 (s, $\left.3 \mathrm{H}, \mathrm{CH}_{3}\right), 7.05-7.11(\mathrm{~m}, 1 \mathrm{H}, \mathrm{Ar}-\mathrm{H} 5), 7.37$ (d, 1H, Ar-H6), 7.47 (s, 1H, vinyl-H), 7.59 (m, 1H, Ar-H4), 7.68 (d, 1H, Ar-H3), 11.51 (s, 1H, NH); MS (EI): m/z (\%)= $187\left(\mathrm{M}^{+}\right)$.

2-[ $N$ "-(2-Oxo-propylidene)-hydrazino]-benzoic acid methyl ester (2c). Yield $2 \mathrm{~g}(90.8 \%)$ as a solid, which was recrystallized from from ethanol as white solid (m.p: 99-101 ${ }^{\circ} \mathrm{C}$ ). Anal. Calcd. for $\mathrm{C}_{11} \mathrm{H}_{12} \mathrm{~N}_{2} \mathrm{O}_{3}$ (220.23): C, 59.99; H, 5.49; N, 12.72. Found: C, 60.20; H, 5.67; N, 12.59. IR $\left(\mathrm{KBr}, \mathrm{cm}^{-1}\right): 3248.3(\mathrm{NH}), 1677.7,1593(\mathrm{CO}) .{ }^{1} \mathrm{H}$ MNR (300 MHz, DMSO-d6): $\delta, \mathrm{ppm}, \delta=2.35$ $\left(\mathrm{s}, 3 \mathrm{H}, \mathrm{CH}_{3}\right), 3.87\left(\mathrm{~s}, 3 \mathrm{H}, \mathrm{OCH}_{3}\right), 7.00-7.06$ (m, 1H, Ar-H5), $7.6(\mathrm{~s}, 1 \mathrm{H}$, vinyl-H), 7.60-7.63 (m, 1H, Ar-H4), 7.76 (d, 1H, Ar-H3), 7.89 (d, 1H, Ar-H6) 11.37 (s, 1H, NH) MS (EI): m/z (\%) = $220\left(\mathrm{M}^{+}\right)$

General method for preparation of $4 \mathbf{b}$ and $4 c$. A mixture of $2 b$ or $2 c(0.01$ mol), ethyl cyanoacetate $(0.01 \mathrm{~mol})$, and ammonium acetate $(2 \mathrm{~g})$ in acetic acid $(20 \mathrm{ml})$ was heated at $200{ }^{\circ} \mathrm{C}$ for $5 \mathrm{~min}$, then left to cool and poured onto water. The solid product obtained was crystallized from ethanol.

2-Cyano-4-[(2-cyano-phenyl)-hydrazono]-3-methyl-but-2-enoic acid ethyl ester (4b). Yield 2.5 g (88.65\%)( m.p:128-130 $\left.{ }^{\circ} \mathrm{C}\right)$. Anal. Calcd. for $\mathrm{C}_{15} \mathrm{H}_{14} \mathrm{~N}_{4} \mathrm{O}_{2}(282.30): \mathrm{C}, 63.82 ; \mathrm{H}, 5.00 ; \mathrm{N}$, 19.85. Found: C, 63.72; H, 4.83; N, 19.62. IR (KBr, cm $\left.{ }^{-1}\right): 3245.2(\mathrm{NH}), 2228.5(\mathrm{CN}), 1722.5$ (CO) ${ }^{1} \mathrm{H}$ MNR (300 MHz, DMSO-d6): $\delta$, ppm $1.28\left(2 \mathrm{t}, 3 \mathrm{H}, \mathrm{CH}_{3}, J=7.2 \mathrm{~Hz}\right), 2.57$ (s, 3H, $\left.\mathrm{CH}_{3}\right)$, 4.27 ( 2 q, 2H, $\mathrm{CH}_{2}, J=7.2 \mathrm{~Hz}$ ), 7.08-7.71 (m, 4H, Ar-H), 8.44 (s, $0.48 \mathrm{H}$, vinyl -H), $9.11(\mathrm{~s}, 0.52$ $\mathrm{H}$, vinyl-H), 11.99 (s, 1H, NH). MS (EI): m/z (\%) = $282\left(\mathrm{M}^{+}\right)$.

2-[ $N$ "-(3-cyano-3-ethoxycarbonyl-2-methyl-allylidene)-hydrazino]-benzoic acid methyl ester (4c). Yield $2.72 \mathrm{~g}$ (86.26\%) (m.p: 142-144 ${ }^{\circ} \mathrm{C}$ ). Anal. Calcd. for $\mathrm{C}_{16} \mathrm{H}_{17} \mathrm{~N}_{3} \mathrm{O}_{4}$ (315.33): C, 60.94; H, 5.43; N, 13.33. Found: C, 60.82; H, 5.62; N, 13.61. IR (KBr, cm $\left.{ }^{-1}\right): 3446.8(\mathrm{NH})$, $2211.1(\mathrm{CN}), 1718,1681$ (CO). ${ }^{1} \mathrm{H}$ MNR (300 MHz, DMSO-d6): $\delta$, ppm 1.29 (2 t, 3H, $\mathrm{CH}_{3}, J=$ $7.2 \mathrm{~Hz}), 2.57\left(\mathrm{~s}, 3 \mathrm{H}, \mathrm{CH}_{3}\right), 3.89\left(\mathrm{~s}, 3 \mathrm{H}, \mathrm{OCH}_{3}\right), 4.25\left(2 \mathrm{q}, 2 \mathrm{H}, \mathrm{CH}_{2}, J=7.2 \mathrm{~Hz}\right), 7.03-7.89$ (m, $4 \mathrm{H}, \mathrm{Ar}-\mathrm{H}), 8.42(\mathrm{~s}, 0.47 \mathrm{H}$, vinyl-H), $9.00(\mathrm{~s}, 0.53$, vinyl-H), 11.75 (s, 1H, NH). MS (EI): m/z $(\%)=315\left(\mathrm{M}^{+}\right)$.

Preparation of 2-[N"-(3,3-dicyano-2-methyl-allylidene)-hydrazino]-benzoic acid methyl ester (4d). A mixture of 2c (10 mmol), and malononitrile was refluxed in ethanol $(20 \mathrm{ml}) \mathrm{in}$ presence of piperidine for $5 \mathrm{~min}$. The solvent was evaporated under vacuum and the crude product was collected and crystallized from ethanol / Dioxan.

Yield 2.32 g (86.48\%); m.p: 212-214 ${ }^{\circ} \mathrm{C}$. Anal. Calcd. for $\mathrm{C}_{14} \mathrm{H}_{12} \mathrm{~N}_{4} \mathrm{O}_{2}$ (268.28): C, 62.68; H, 4.51; N, 20.88. Found: C, 62.42; H, 4.72; N, 20.61. IR (KBr, cm $\left.{ }^{-1}\right): 3231.4(\mathrm{NH}), 2218.9(\mathrm{CN})$, 1691.9 (CO). ${ }^{1} \mathrm{H}$ MNR (300 MHz, DMSO-d6): $\delta$, ppm 2.48(s, 3H, $\left.\mathrm{CH}_{3}\right), 3.89\left(\mathrm{~s}, 3 \mathrm{H}, \mathrm{OCH}_{3}\right)$, 7.10-7.90 (m, 4H, Ar-H), 8.40 (s, 1H, vinyl-H), 11.85 (s, 1H, NH) ${ }^{13} \mathrm{C}$ MNR (300 MHz, DMSOd6): $\delta$, ppm $16.88\left(\mathrm{CH}_{3}\right), 52.34\left(\mathrm{OCH}_{3}\right), 79.87(\mathrm{C}-\mathrm{CN}), 112.67,113.76(\mathrm{CN}), 113.96,115.49$, 
$122.05,130.84,134.54,135.44$ (CH-Ar), 143.66 (CH-vinyl), $166.79\left(\mathrm{C}^{\left.-\mathrm{CH}_{3}\right)}, 168.07\right.$ $\left(\mathrm{COOCH}_{3}\right)$. MS (EI): $\mathrm{m} / \mathrm{z}(\%)=268\left(\mathrm{M}^{+}\right)$.

General method for preparation of 5a,b. A mixture of each of $2 \mathrm{a}$ or $2 \mathrm{~b}(0.01 \mathrm{~mol})$, ethyl cyanoacetate $(0.01 \mathrm{~mol})$, and ammonium acetate $(2 \mathrm{~g})$ in acetic acid $(20 \mathrm{ml})$ was heated under reflux for 3 hours, then left to cool and poured onto water. The solid product obtained was crystallized from ethanol.

4-Methyl-6-oxo-1-phenyl-1,6-dihydropyridazine-5-carbonitrile (5a). Yield $1.82 \mathrm{~g} \mathrm{(86.16 \% )}$ (m.p: $158-160{ }^{\circ} \mathrm{C}$ ) . Anal. Calcd. for $\mathrm{C}_{12} \mathrm{H}_{9} \mathrm{~N}_{3} \mathrm{O}$ (211.23): C, 68.24; H, 4.29; N, 19.89. Found: C, 68.16; H, 4.14; N, 20.01. IR (KBr, cm $\left.{ }^{-1}\right): 2233(\mathrm{CN}), 1659.4(\mathrm{CO}) .{ }^{1} \mathrm{H}$ MNR (300 MHz, DMSOd6): $\delta$, ppm 2.47 (s, $\left.3 \mathrm{H}, \mathrm{CH}_{3}\right), 7.54-7.55$ (m. $\left.5 \mathrm{H}, \mathrm{Ar}-\mathrm{H}\right), 8.25$ (s, $1 \mathrm{H}$, pyridazine-H). ${ }^{13} \mathrm{C}$ MNR (300 MHz, DMSO-d6): $\delta$, ppm $19.12\left(\mathrm{CH}_{3}\right), 114.06(\mathrm{C}-\mathrm{CN}), 114.48(\mathrm{CN}), 126.59,126.83$,

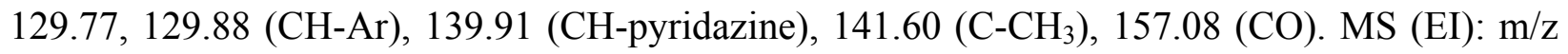
$(\%)=211\left(\mathrm{M}^{+}\right)$.

1-(2-Cyano-phenyl)-4-methyl-6-oxo-1,6-dihydropyridazine-5-carbonitrile (5b). Yield $2.0 \mathrm{~g}$ (84.47\%) (m.p: 170-172 ${ }^{\circ}$ C). Anal. Calcd. for $\mathrm{C}_{13} \mathrm{H}_{8} \mathrm{~N}_{4} \mathrm{O}$ (236.23): C, 66.10; H, 3.41; N, 23.72. Found: C, 66.26; H, 3.54; N, 23.85. IR (KBr, cm $\left.{ }^{-1}\right): 2232.8(\mathrm{CN}), 1665.8(\mathrm{CO}) .{ }^{1} \mathrm{H}$ MNR (300 MHz, DMSO-d6): $\delta$, ppm 2.52 (s, 3H, $\mathrm{CH}_{3}$ ), 7.71-7.76 (m, 2H, Ar-H3,4), 7.93 (m, 1H, Ar-H5), 8.09 (d, 1H, Ar-H6), 8.34 (s, 1H, pyrimidine-H). MS (EI): $\mathrm{m} / \mathrm{z}(\%)=236\left(\mathrm{M}^{+}\right)$..

Preparation of 3-methyl-6-oxo-6,11-dihydropyridazino[1,6-a]quinazoline-4-carbonitrile (6) A mixture of $2 \mathrm{c}(10 \mathrm{mmol})$, and malononitrile was refluxed in ethanol $(20 \mathrm{ml})$ in presence of piperidine for $30 \mathrm{~min}$. The solvent was evaporated under vacuum and the crude product was collected and crystallized from Dioxan.

Yield 2.1 g (88.9\%) (m.p: $280-282{ }^{\circ}$ C). Anal. Calcd. for $\mathrm{C}_{13} \mathrm{H}_{8} \mathrm{~N}_{4} \mathrm{O}$ (236.23): C, 66.10; H, 3.41; $\mathrm{N}, 23.72$. Found: $\mathrm{C}, 66.23 ; \mathrm{H}, 3.52 ; \mathrm{N}, 23.61$. IR $\left(\mathrm{KBr}, \mathrm{cm}^{-1}\right): 2234.9(\mathrm{CN}), 1655(\mathrm{CO}) .{ }^{1} \mathrm{H} \mathrm{MNR}$ (300 MHz, DMSO-d6): $\delta$, ppm $2.61\left(\mathrm{~s}, 3 \mathrm{H}, \mathrm{CH}_{3}\right), 7.72-8.39(\mathrm{~m}, 4 \mathrm{H}, \mathrm{Ar}-\mathrm{H}), 8.78(\mathrm{~s}, 1 \mathrm{H}$, pyridazine-H). MS (EI): $\mathrm{m} / \mathrm{z}(\%)=236\left(\mathrm{M}^{+}\right)$.

General procedure for preparation of phthalazines 9a-f. Methode A. A mixture of 4 (or 5) $(10 \mathrm{mmol})$, and benzylidene derivatives 7 was refluxed in ethanol $(20 \mathrm{ml})$ in presence of piperidine for $2 \mathrm{~h}$. The solvent was evaporated under vacuum and the crude product was collected and crystallized from ethanol.

Methode B. A mixture of 4 (or 5) $(10 \mathrm{mmol})$, and benzylidene derivatives 7 (10 mmol) in pyridine $(2 \mathrm{ml})$ was irradiated in microwave oven for two minutes, then poured onto water and acidified with dilute hydrochloric acid. The solid product obtained was crystallized from ethanol.

5-amino-4-oxo-3,4-dihydro-phthalazine-6-carbonitrile (9a). Yield $3.20 \mathrm{~g}$ (80.40\%); m.p: 288$290{ }^{\circ}$ C. Anal. Calcd. for $\mathrm{C}_{21} \mathrm{H}_{14} \mathrm{~N}_{4} \mathrm{O}$ (338.37): C, 74.54; H, 4.17; N, 16.56. Found: C, 74.62; H, 4.24; N, 16.71. IR ( $\left.\mathrm{KBr}, \mathrm{cm}^{-1}\right): 3453.4,3300.4\left(\mathrm{NH}_{2}\right), 2207.1(\mathrm{CN}), 1657.2(\mathrm{CO}) .{ }^{1} \mathrm{H}$ MNR $(300$ $\mathrm{MHz}, \mathrm{CDCl}_{3}$ ): $\delta$, ppm 6.92 (s, $\left.1 \mathrm{H}, \mathrm{Ar}-\mathrm{H}\right), 7.28$ (br s, $\left.2 \mathrm{H}, \mathrm{NH}_{2}\right), 7.43-7.64(\mathrm{~m}, 10 \mathrm{H}, \mathrm{Ar}-\mathrm{H}) 8.17$ (s, $1 \mathrm{H}$, pyridazine-H). ${ }^{13} \mathrm{C}$ MNR (300 MHz, $\left.\mathrm{CDCl}_{3}\right): \delta$, ppm $96.48(\mathrm{C}-\mathrm{CN}), 110.48(\mathrm{CN}), 113.85$, $117,126.4,128.91,129.06,129.36,129.05,129.56,130.23,134.25,138.17,139.25,141.69$ (CH-Ar), 151.51(C-NH $)_{2}, 154.21\left(\mathrm{CH}-\right.$ pyridazine), $161.02(\mathrm{CO})$. MS (EI): $\mathrm{m} / \mathrm{z}(\%)=338\left(\mathrm{M}^{+}\right)$. 
5-Amino-4-oxo-3,7-diphenyl-3,4-dihydro-phthalazine-6-carboxylic acid ethyl ester (9b). Yield 33.2 g (86.1\%) (m.p: 112-114 ${ }^{\circ} \mathrm{C}$ ). Anal. Calcd. for $\mathrm{C}_{23} \mathrm{H}_{19} \mathrm{~N}_{3} \mathrm{O}_{3}$ (385.43): C, 71.68; H, 4.97; N, 10.90. Found: C, 71.62; H, 4.92; N, 10.81. IR (KBr, cm $\left.{ }^{-1}\right): 3432.3,3293.6\left(\mathrm{NH}_{2}\right)$, 1688.1, 1640.6 (CO). ${ }^{1} \mathrm{H}$ MNR (300 MHz, DMSO-d6): $\delta$, ppm 0.77 (t, 3H, $\mathrm{CH}_{3}, J=7.2 \mathrm{~Hz}$ ), 3.96 (q, 2H, $\mathrm{CH}_{2}, J=7.2 \mathrm{~Hz}$ ), 6.97 (s, 1H, Ar-H), 7.32-7.62 (m, 10H, Ar-H), 7.84 (br s, 2H, $\left.\mathrm{NH}_{2}\right), 8.34$ (s, 1H, pyridazine-H). ${ }^{13} \mathrm{C}$ MNR (300 MHz, DMSO-d6): $\delta$, ppm $13.11\left(\mathrm{CH}_{3}\right), 60.69$ $\left(\mathrm{CH}_{2}\right)$, 113.7(C-COOEt), 125.73, 126.19, 127.64, 127.88, 128.33, 128.55, 128.64, 132.17, 139.19, 140.95, 141.66 (CH-Ar), 148.12 (C-Ph), $149.75\left(\mathrm{C}-\mathrm{NH}_{2}\right), 160.63$ (CH-pyridazine, 167.42 (CO). MS (EI): m/z (\%)=385 $\left(\mathrm{M}^{+}\right)$.

5-amino-7-(4-chloro-phenyl)-3-(2-cyano-phenyl)-4-oxo-3,4-dihydro-phthalazine-6carbonitrile (9c). Yield 3.20 g (80.4\%); m.p: 288-290 ${ }^{\circ} \mathrm{C}$. Anal. Calcd. for $\mathrm{C}_{22} \mathrm{H}_{12} \mathrm{ClN}_{5} \mathrm{O}$ (397.83): C, 66.42; H, 3.04; N, 17.60. Found: C, 66.53; H, 3.16; N, 17.63. IR ( $\left.\mathrm{KBr}, \mathrm{cm}^{-1}\right)$ : 3471.9, 3332.1 $\left(\mathrm{NH}_{2}\right), 2208.8(\mathrm{CN}), 1656.1$ (CO). ${ }^{1} \mathrm{H}$ MNR (300 MHz, DMSO-d6): $\delta$, ppm 7.18 (s, 1H, Ar-H), 7.63-7.95 (m, 8H, Ar-H), 8.07 (s, 2H, NH 2$), 8.52$ (s, 1H, pyridazine-H). MS (EI): $\mathrm{m} / \mathrm{z}(\%)=397\left(\mathrm{M}^{+}\right)$.

2-[8-Amino-6-(4-chloro-phenyl)-7-cyano-1-oxo-1H-phthalazin-2-yl]-benzoic acid methyl ester (9d). Yield $3.52 \mathrm{~g}$ (81.7\%) (m.p: 295-297 ${ }^{\circ} \mathrm{C}$ ). Anal. Calcd. for $\mathrm{C}_{23} \mathrm{H}_{15} \mathrm{ClN}_{4} \mathrm{O}_{3}$ (430.85): C, 64.12; H, 3.51; N, 13. Found: C, 64.25; H, 3.42; N, 12.92. IR $\left(\mathrm{KBr}, \mathrm{cm}^{-1}\right): 3437.7,3356.2\left(\mathrm{NH}_{2}\right)$, $2213.9(\mathrm{CN}), 1686.8,1651.3$ (CO). ${ }^{1} \mathrm{H}$ MNR (300 MHz, DMSO-d6): $\delta$, ppm 3.87 (s, 3H, $\mathrm{CH}_{3}$ ), 6.73 (br s, $\left.2 \mathrm{H}, \mathrm{NH}_{2}\right), 6.90-7.87(\mathrm{~m}, 9 \mathrm{H}, \mathrm{Ar}-\mathrm{H}), 8.31(\mathrm{~s}, 1 \mathrm{H}$, pyridazine-H). MS (EI): m/z $(\%)=$ $430\left(\mathrm{M}^{+}\right)$.

5-Amino-3-(2-methoxycarbonyl-phenyl)

-4-oxo-7-phenyl-3,4-dihydro-phthalazin-6carboxylic acid ethyl ester (9e). Yield $3.51 \mathrm{~g}$ (79.15\%) (m.p: 158-160 $\left.{ }^{\circ} \mathrm{C}\right)$. Anal. Calcd. for $\mathrm{C}_{25} \mathrm{H}_{21} \mathrm{~N}_{3} \mathrm{O}_{5}$ (443.46): C, 67.71; H, 4.77; N, 9.48. Found: C, 67.63; H, 4.62; N, 9.32. IR (KBr, $\mathrm{cm}^{-1}$ ): 3459.8, 3310.1( $\left.\mathrm{NH}_{2}\right), 1728,1691.5,1641.5$ (CO). ${ }^{1} \mathrm{H}$ MNR (300 MHz, DMSO-d6): $\delta$, ppm 0.75 (t, 3H, $\left.\mathrm{CH}_{3}, J=7.2 \mathrm{~Hz}\right), 3.66\left(\mathrm{~s}, 3 \mathrm{H}, \mathrm{CH}_{3}\right), 3.95$ (q, 2H, $\left.\mathrm{CH}_{2}, J=7.2 \mathrm{~Hz}\right), 7.01$ (s, 1H, Ar-H), 7.34 (br s, 2H, NH 2$), 7.43-7.97(\mathrm{~m}, 9 \mathrm{H}, \mathrm{Ar}-\mathrm{H}), 8.36(\mathrm{~s}, 1 \mathrm{H}$, pyridazine-H). MS (EI): m/z $(\%)=443\left(\mathrm{M}^{+}\right)$.

2-[8-Amino-6-(4-chloro-phenyl)-7-cyano-1-imino-1H-phthalazin-2-yl]-benzoic acid methyl ester (9f). Yield $3.31 \mathrm{~g}$ (77\%) (m.p: $320{ }^{\circ} \mathrm{C}$ ). Anal. Calcd. for $\mathrm{C}_{23} \mathrm{H}_{16} \mathrm{ClN}_{5} \mathrm{O}_{2}$ (429.87): C, 64.27; H, 3.75; N, 16.29. Found: C, 64.44; H, 3.86; N, 16.25. IR (KBr, cm $\left.{ }^{-1}\right): 3432.3(\mathrm{NH}), 3353$, $3246.3\left(\mathrm{NH}_{2}\right), 2212.9(\mathrm{CN}), 1685.5$ (CO). ${ }^{1} \mathrm{H}$ MNR (300 MHz, DMSO-d6): $\delta$, ppm 3.88 (s, 3H, $\left.\mathrm{CH}_{3}\right), 6.74\left(\mathrm{~m}, 11 \mathrm{H}, \mathrm{Ar}-\mathrm{H}\right.$ and $\left.\mathrm{NH}_{2}\right), 8.32(\mathrm{~s}, 1 \mathrm{H}$, pyridazine-H), $11.41(\mathrm{~s}, 1 \mathrm{H}, \mathrm{NH}) ; \mathrm{MS}(\mathrm{EI}) \mathrm{m} / \mathrm{z}$ $(\%)=429\left(\mathrm{M}^{+}\right)$.

General procedures for preparation of triaza-phenanthrenes 13a-c and 16. Methode A. A mixture of azaenamine $2 \mathrm{~b}$ or $2 \mathrm{c}(10 \mathrm{mmol})$, and benzylidene derivatives $6 \mathrm{a}-\mathrm{c}$ was refluxed in pyridine $(20 \mathrm{ml})$ for $2 \mathrm{~h}$., then poured onto water and acidified with dilute hydrochloric acid. The solid product obtained was crystallized from ethanol.

Methode B. A solution of each of $2 \mathrm{~b}$ or $2 \mathrm{c}(10 \mathrm{mmol})$ was treated with the benzylidene $7 \mathrm{a}-\mathrm{c}(10$ 
mmol) in pyridine $(2 \mathrm{ml})$ was irradiated in microwave oven for two minutes, then poured onto water and acidified with dilute hydrochloric acid. The solid product obtained was crystallized from ethanol.

2-Acety;-6-amino-3-(4-chlorophenyl)-3,11-dihydro-pyridazino[1,6-a]quinazoline-4carbonitrile (13a). Yield 3.15 g (83.82\%); m.p: 230-232 ${ }^{\circ} \mathrm{C}$. Anal. Calcd. for $\mathrm{C}_{20} \mathrm{H}_{14} \mathrm{ClN}_{5} \mathrm{O}$ (375.82): C, 63.92; H, 3.75; N, 18.63. Found: C, 63.63; H, 3.69; N,3.84. IR (KBr, $\left.\mathrm{cm}^{-1}\right): 3435.8$, $3425.1\left(\mathrm{NH}_{2}\right), 2223(\mathrm{CN}), 1687(\mathrm{CO}) .{ }^{1} \mathrm{H}$ MNR (300 MHz, DMSO-d6): $\delta$, ppm 2.47 (s, 3H, $\left.\mathrm{CH}_{3}\right), 4.9$ (s, 1H, pyridazine-H), 7.18(s, 2H, $\left.\left.\mathrm{NH}_{2}\right), 7.29-8.15(\mathrm{~m}, 8 \mathrm{H}, \mathrm{Ar}-\mathrm{H})\right)$ ) ${ }^{13} \mathrm{C} \mathrm{MNR}(300$ $\mathrm{MHz}, \mathrm{DMSO}-\mathrm{d} 6): \delta$, ppm $25.21\left(\mathrm{CH}_{3}\right), 37.28(\mathrm{CH}$ pyridazine $), 67.65(\mathrm{C}-\mathrm{CN}), 112.08(\mathrm{CN})$, $114.44,120.48,124.15,124.72,128.89,129.25,132.12,134.43,139.94,141.37$ (CH-Ar), 144.68(C-COCH 3$), 147.30$ (C6-pyridazine), 156.39(C- $\left.\mathrm{NH}_{2}\right), 195.72(\mathrm{CO}) . \mathrm{MS}(\mathrm{EI}): \mathrm{m} / \mathrm{z}(\%)=$ $375\left(\mathrm{M}^{+}\right)$.

Ethyl-2-Acety-6-amino-3-phenyl-3,11-dihydro-pyridazino[1,6-a]quinazoline-4-carboxylate (13b). Yield 3.15 g (83.82\%) (m.p: 194-196 ${ }^{\circ} \mathrm{C}$ ). Anal. Calcd. for $\mathrm{C}_{22} \mathrm{H}_{20} \mathrm{~N}_{4} \mathrm{O}_{3}$ (388.43): C,68.03; H, 5.19; N, 14.42. Found: C, 68.22; H, 5.23; N,14.36. IR (KBr, $\left.\mathrm{cm}^{-1}\right): 3490.9,3375.2\left(\mathrm{NH}_{2}\right)$, 1681.8 (CO). ${ }^{1} \mathrm{H}$ MNR (300 MHz, DMSO-d6): $\delta$, ppm 1.19 (t, 3H, $\mathrm{CH}_{3}, J=6.9 \mathrm{~Hz}$ ), 2.53 (s, 3H, $\left.\mathrm{CH}_{3}\right), 4.06\left(\mathrm{q}, 2 \mathrm{H}, \mathrm{CH}_{2}, J=6.9 \mathrm{~Hz}\right), 5.34\left(\mathrm{~s}, 1 \mathrm{H}\right.$, pyridazine-H), $7.06\left(\mathrm{~s}, 2 \mathrm{H}, \mathrm{NH}_{2}\right), 7.20-8.06(\mathrm{~m}$, $9 \mathrm{H}, \mathrm{Ar}-\mathrm{H})$. MS (EI): $\mathrm{m} / \mathrm{z}(\%)=388\left(\mathrm{M}^{+}\right)$.

2-Acety;-6-amino-4-benzoyl-3-phenyl-3,11-dihydro-pyridazino[1,6-a]quinazoline (13c)

Yield 3.12 g (74.20\%) (m.p: 222-224 ${ }^{\circ} \mathrm{C}$ ). Anal. Calcd. for $\mathrm{C}_{26} \mathrm{H}_{20} \mathrm{~N}_{4} \mathrm{O}_{2}$ (420.47): C,74.27; H, 4.79; N, 13.32. Found: C, 74.15; H, 4.83; N,13.26. IR (KBr, cm $\left.{ }^{-1}\right): 3444.9,3332.9\left(\mathrm{NH}_{2}\right)$, 1686.6, 1637.7 (CO). ${ }^{1} \mathrm{H}$ MNR (300 MHz, DMSO-d6): $\delta$, ppm 2.60 (s, 3H, $\mathrm{CH}_{3}$ ), 5.37 (s, 1H, pyridazine-H), 7.10 (s, 2H, NH$\left.)_{2}\right) 7.15-8.04$ (m, 14H, Ar-H); MS (EI): m/z (\%)= $420\left(\mathrm{M}^{+}\right) .3-$

Ethyl-2-Acety-6-oxo-3-phenyl-3,5,6,11-tetrahydro-pyridazino[1,6-a]quinazoline-4carboxylate (16). Yield 3.12 g (80.12\%) (m.p: 206-208 ${ }^{\circ} \mathrm{C}$ ). Anal. Calcd. for $\mathrm{C}_{22} \mathrm{H}_{19} \mathrm{~N}_{3} \mathrm{O}_{4}$ (389.41): C,67.86; H, 4.92; N, 10.79. Found: C, 67.78; H, 4.83; N,10.67. IR (KBr, cm $\left.{ }^{-1}\right): 3432$ $(\mathrm{NH}), 1718,1695.5,1639$ (CO). ${ }^{1} \mathrm{H}$ MNR (300 MHz, DMSO-d6): $\delta$, ppm 1.15 (t, 3H, $\mathrm{CH}_{3}, J=$ $7.2 \mathrm{~Hz}$ ), $2.48\left(\mathrm{~s}, 3 \mathrm{H}, \mathrm{CH}_{3}\right), 4.09$ (q, 2H, $\left.\mathrm{CH}_{2}, J=7.2 \mathrm{~Hz}\right), 5.14$ (s, 1H, pyridazine-H), 7.18 (s, $\left.2 \mathrm{H}, \mathrm{NH}_{2}\right), 7.24-8.01(\mathrm{~m}, 9 \mathrm{H}, \mathrm{Ar}-\mathrm{H}), 11.66(\mathrm{~s}, 1 \mathrm{H}, \mathrm{NH}) . \mathrm{MS}(\mathrm{EI}): \mathrm{m} / \mathrm{z}(\%)=389\left(\mathrm{M}^{+}\right)$.

\section{References}

1. Tamayo, N.; Liao, L.; Goldberg, M.; Powers, D.; Tudor, Y-Y.; Yu, V.; Wong, L.M.; Henkle, B.; Middleton, S.; Syed R.; Harvey, T.; Jang, G.; Hungate, R.; and Dominguez, C. Bioorganic \& Medicinal chemistry Letters 2005, 15, 2409.

2. McIntyre, C.J.; Ponticello, G.S.; Liverton, N.J.; O’Keefe, S.J.; O’Neill, E.A.; Pang, M.; Schwartz, C.D. and Claremon, D.A. Bioorg. Med. Chem. Lett. 2002, 12, 689.

3. Nagawade,R. R.; Khanna, V. V.; Bhagwat S. S.; Shinde D. B. European Journal of Medicinal Chemistry 2005, 40, 1325. 
4. Lee, S. J.; Lee, J. S.; Hwang, K.-J.; Kim, Y. K.; Park, N. G.; Shin, E. J; and Lee, S. H. Current applied physics 2005, 5, 43.

5. MERCK \& CO INC (US); LEBSACK ALECD (US); MUNOZ BENITO (US); PRACITTO RICHARD (US); VENKATRAMAN SHANKAR (US); WANG BOWEI (US); GUNZNER JANET (US), European, 2005, patent number (WO2005041971), priority number (US20030513046p 20031021.

6. Lee, S.-G.; Kim, J.-J.; Kim, H.-K.; Kweon, D.-H.; Kang, Y.-J.; Cho, S.-D.; Kim, S.-K.; and Yoon, Y.-J. Current Organic Chemistry 2004, 8, 1463.

7. Sotelo, E.; Ravina, E.; Synth. Commun. 2002, 32, 1675.

8. Giovannoni, M.P.; Dal Piaz, V.; Kwon, B.M.; Kim, M.K.; Kim, Y.K.; Toma, L.; Barlocco, D.; Bernini, F.; Canavesi, M., J. Med. Chem. 2001, 44, 1473.

9. Coelho, A.; Sotelo, E.; Novoa, H.; Peeters, O. M.; Blaton, N.; and Ravina, E.; Tetrahedron Letters 2004, 45, 3459.

10. Sotelo, E.; Fraiz, N.; Yañez, M.; Brea, J.M.; Laguna, R.; Cano, E. and Raviña, E., Bioorg. Med. Chem. Let. 2002,. 10, 1575.

11. Elghandour, A. H. H.; Ibrahim, M. K. A.; Hafez, I.S.A.; Elnagdi, M. H., Org. Prep.Proced. Int. 1993, 25, 293.

12. Behbehani, H.; Abdel-Khalik, M. M.; Elnagdi, M. H., Org. Prep.Proced. Int. 1999, 31, 551.

13. Ghozlan,S. A. S.; Abdelhamid, I. A.; Hassaneen, H. M.; and Elnagdi, M. H. Accepted for publication in Journal Hetrocycl. Chem. 2006.

14. Hassaneen, H. M. E.; Abdallah, T. A.; Hassaneen, H. M.; Elnagdi, M. H., Journal of Chem. Res. 2005, 729.

15. Al-Saleh, B.; Helmy, N. M.; Al-Apasery, M.; Elnagdi, M. H. Journal Heterocycl. Chem. 2006, in press.

16. Ancel, J. E.; El Kaiim, L.; Gadras, A.; Grimaud, L.; Jana, N. K. Tetrahedron Letters 2002, 43,8319

17. Atlan, V.; Bienayme, H.; El-Kaim, L.; Majee, A. Chem. Commmun. 2000, 1585.

18. Buff, H.; Kucklander, U. Tetrahedron 2000, 56, 5137.

19. Smith, M. B.; , Organic Synthesis, $2^{\text {nd }}$ Edn; Mc Graw Hill: London, 2002. 